\title{
Drusen characteristics of type 2 macular neovascularization in age-related macular degeneration
}

\author{
Daniel Ahmed ${ }^{1,2+}$, Martin Stattin ${ }^{1,2+}$, Anna-Maria Haas ${ }^{1,2}$, Alexandra Graf ${ }^{3}$, Katharina Krepler ${ }^{1,2}$ and \\ Siamak Ansari-Shahrezaei ${ }^{1,2,4^{*}}$ (D)
}

\begin{abstract}
Background: Type 2 macular neovascularization (MNV) is supposed to be a rare condition in age-related macular degeneration (AMD). The main purpose of this study was to assess accompanying factors of type 2 MNV in AMD.

Methods: Retrospective data analysis of eyes previously diagnosed with neovascular AMD in a tertiary eye care center (Medical Retina Unit, Rudolf Foundation Hospital, Vienna, Austria) between June 2008 and December 2017. Drusen subtypes, fibrosis, atrophy and subfoveal choroidal thickness (SFCT) of both eyes in patients with type 2 MNV lesions were categorized based on multimodal imaging.

Results: Type 2 MNV was diagnosed in 27 (3.2\%) of 835 eyes (749 patients). Drusen characteristics in type 2 MNV were observed as followed: drusen $<63 \mu \mathrm{m}$ in 2 eyes (7.4\%), drusen $\geq 63 \mu \mathrm{m}$ in 10 eyes (37\%), subretinal drusenoid deposits (SDD) in 8 eyes (29.6\%), cuticular drusen in 2 eye (7.4\%) and no drusen were evident in 10 eyes (37\%). Drusen distribution in 23 fellow eyes was detected as followed: drusen $<63 \mu \mathrm{m}$ in 2 eyes (8.7\%), drusen $\geq 63 \mu \mathrm{m}$ in 9 eyes (39.1\%), SDD in 5 eyes (21.7\%), cuticular drusen in 1 eye (4.3\%) and no drusen were evident in 9 eyes (39.1\%). Mean SFCT was $140 \pm 49 \mu \mathrm{m}$ in affected eyes and $152 \pm 41 \mu \mathrm{m}$ in the fellow eyes. Patients with drusen or SDD were significantly younger (mean $70.88 \pm 6.85, p=0.04$ ) than patients without deposits (mean $77.40 \pm 5.74$ ).

Conclusions: Type 2 MNV remains a rare entity in AMD. It was frequently seen in the absence of drusen, a hallmark of AMD. These findings contribute to the heterogeneity of phenotypes related to pure type 2 lesions.
\end{abstract}

Keywords: Age-related macular degeneration, Type 2 macular neovascularization, Drusen, Subretinal drusenoid deposits

\section{Background}

Early and intermediate age-related macular degeneration (AMD) are defined by the existence of drusen, pigmentary abnormalities or extrafoveal atrophy [1,2]. Various types of drusen such as soft, hard, cuticular or subretinal

\footnotetext{
* Correspondence: siamak.ansari-shahrezaei@wienkav.at; siamak.ansarishahrezaei@wienkav.at

${ }^{\dagger}$ Daniel Ahmed and Martin Stattin contributed equally to this work. 'Karl Landsteiner Institute for Retinal Research and Imaging, Vienna, Austria ${ }^{2}$ Department of Ophthalmology, Rudolf Foundation Hospital, Juchgasse 25, 1030 Vienna, Austria

Full list of author information is available at the end of the article
}

drusenoid deposits (SDD) - also referred to as reticular pseudodrusen - confirm with the diagnosis of AMD [3]. Drusen are typically located between the retinal pigment epithelium (RPE) and the Bruch's membrane, whereas SDD are found in the subretinal space [4-7]. Small drusen $(<63 \mu \mathrm{m})$ are considered normal aging changes, while medium $(\geq 63-<125 \mu \mathrm{m})$ to large drusen $(\geq 125 \mu \mathrm{m})$ are associated with an increased risk for the development of late stage disease. Advanced AMD refers to either neovascular AMD (nAMD) or subfoveal geographic atrophy (GA) [1]. Spaide et al. suggested the

(c) The Author(s). 2020, corrected publication 2020. Open Access This article is licensed under a Creative Commons Attribution 4.0 International License, which permits use, sharing, adaptation, distribution and reproduction in any medium or format, as long as you give appropriate credit to the original author(s) and the source, provide a link to the Creative Commons licence, and indicate if changes were made. The images or other third party material in this article are included in the article's Creative Commons licence, unless indicated otherwise in a credit line to the material. If material is not included in the article's Creative Commons licence and your intended use is not permitted by statutory regulation or exceeds the permitted use, you will need to obtain permission directly from the copyright holder. To view a copy of this licence, visit http://creativecommons.org/ licenses/by/4.0/. The Creative Commons Public Domain Dedication waiver (http://creativecommons.org/publicdomain/zero/1. 0/) applies to the data made available in this article, unless otherwise stated in a credit line to the data. 
term macular neovascularization (MNV) more adequate to sum up all neovascular entities in AMD including type $3[8,9]$. Therefore, the author proposed a new classification system encompassing extracellular deposits as well as the subfoveal choroidal thickness (SFCT) to integrate all aspects of the disease. Type $1 \mathrm{MNV}$ originates from the choroid and remains exclusively underneath the RPE. Polypoidal choroidal vasculopathy has recently been described as aneurysmal neovascularization type 1 and represents a subtype of this entity [10]. A mixed type neovascularization is composed of new choroidal vessels growing in more than one layer. A vascular network only present in the subretinal space is referred to as type $2 \mathrm{MNV}$. In contrast, type 3 neovascularization also called retinal angiomatous proliferation (RAP) - reflects a distinct form of nAMD with an intraretinal origin and hence different pathophysiology [11].

While type $1 \mathrm{MNV}$ is the most common subtype in nAMD followed by type 3 and mixed type MNV, the appearance of type $2 \mathrm{MNV}$ is a rare condition $[12,13]$. So far, drusen characteristics were mainly investigated in type $1 \mathrm{MNV}[1,2,14-16]$. Type 2 and mixed type MNV with a preponderance towards type 2 - formerly known as classic or predominantly classic choroidal neovascularization - were observed in $11.5-24.6 \%$ in the era of FA alone [17-20]. SD-OCT imaging led to a deeper knowledge of the neovascular anatomy as well as the aggregation of extracellular debris [11]. A combination of FA and OCT reduced the percentage of type $2 \mathrm{MNV}$ to $0.8-9 \%$ according to the most recent studies with a focus on deposits and choroidal thickness in the latter one $[12,13]$.

Accompanying factors of type $2 \mathrm{MNV}$ remain still unclear. The main purpose of this study was to gain a better understanding of the characteristics associated with type $2 \mathrm{MNV}$ in AMD based on multimodal imaging.

\section{Methods}

\section{Patient selection}

This retrospective observational case series included treatment- naive patients with nAMD, diagnosed in our tertiary retina center (Medical Retina Unit, Department of Ophthalmology; Rudolf Foundation Hospital Vienna; Karl Landsteiner Institute for Retinal Research and Imaging) between 2008 and December 2017. The study adhered to the tenets of the Declaration of Helsinki. Patients signed an informed consent to participate anonymously in a clinical study. Consenting patients were initially diagnosed and classified into anatomic subtypes of neovascular lesions based on fundus examination by slit-lamp biomicroscopy (Haag- Streit AG, Bern, Switzerland) and multimodal imaging including nearinfrared fundus reflectance (IR), blue-peak fundus autofluorescence (BAF) images, spectral-domain (SD) optical coherence tomography (OCT), high resolution fluorescein angiography (FA) and indocyanine green angiography (ICGA). Exclusion criteria were neovascularizations of different entities (p.e. idiopathic, myopic, posttraumatic, uveitic, dystrophic or secondary to pachychoroid diseases) as well as a subfoveal atrophy or a fibrosis at the time of recruitment. The fellow eye was examined for additional information on the drusen distribution and also excluded in case of a neovascularization, subfoveal atrophy or fibrosis. Macular neovascularization secondary to age-related degeneration was concluded if deposits or any other causative origin were absent in both eyes.

\section{Image interpretation}

The data records of patients with type $2 \mathrm{MNV}$ were independently analyzed by two medical retina specialists based on all available images. Type $2 \mathrm{MNV}$ was diagnosed in case of an early leakage and the absence of speckled hyperfluoresence in FA [12]. Multiple SD-OCT scans through the lesion area were conducted to assign the layer according to the subtype classification relative to the RPE. If $95 \%$ or more of the lesion was judged by FA and OCT to be type $2 \mathrm{MNV}$, then it was categorized as type $2 \mathrm{MNV}$. ICGA differentiated the neovascular lesion as a well-defined network with a circumscribed halo in early phases from other types like polypoidal lesions or hot spots mandatory for type $3 \mathrm{MNV}$. Moreover, ICGA was helpful to distinguish between neovascularization related to AMD vs. a secondary neovascularization masquerading AMD.

\section{Characteristics}

Greyscale variations in IR images on high resolution angiography and OCT scans indicated the existence of soft drusen (Fig. 1a). SDD commonly appeared as hyporeflective dots (Fig. 1e) or ribbons but could also occur as hyperreflective spots surrounding the perifoveal region, termed midperipheral SDD [21].

In BAF images, soft, hard and cuticular drusen appeared hypoautofluorescent in the center with an annulus of increased fundus autofluorescence [22, 23]. A reticular hypoautofluorescent pattern was typical for SDD [24]. Different staining levels in FA depend on the binding of dye to polar lipids with a higher proportion of fibronectin in contrast to neutral lipids with little adherence [25]. Soft drusen showed mild hyperfluorescence on FA (Fig. 1b, f) but hypofluorescence on ICGA (Fig. 1c), while hard drusen were often hyperfluorescent in both dye applications. SDD were hypofluorescent or not visible on FA and ICGA (Fig. 1b, c, f) [6, 7, 26-28]. The hyperfluorescent "starry sky" appearance on FA was considered as typical for cuticular drusen (Fig. 2a, c) [29]. OCT images were investigated to locate the layer 


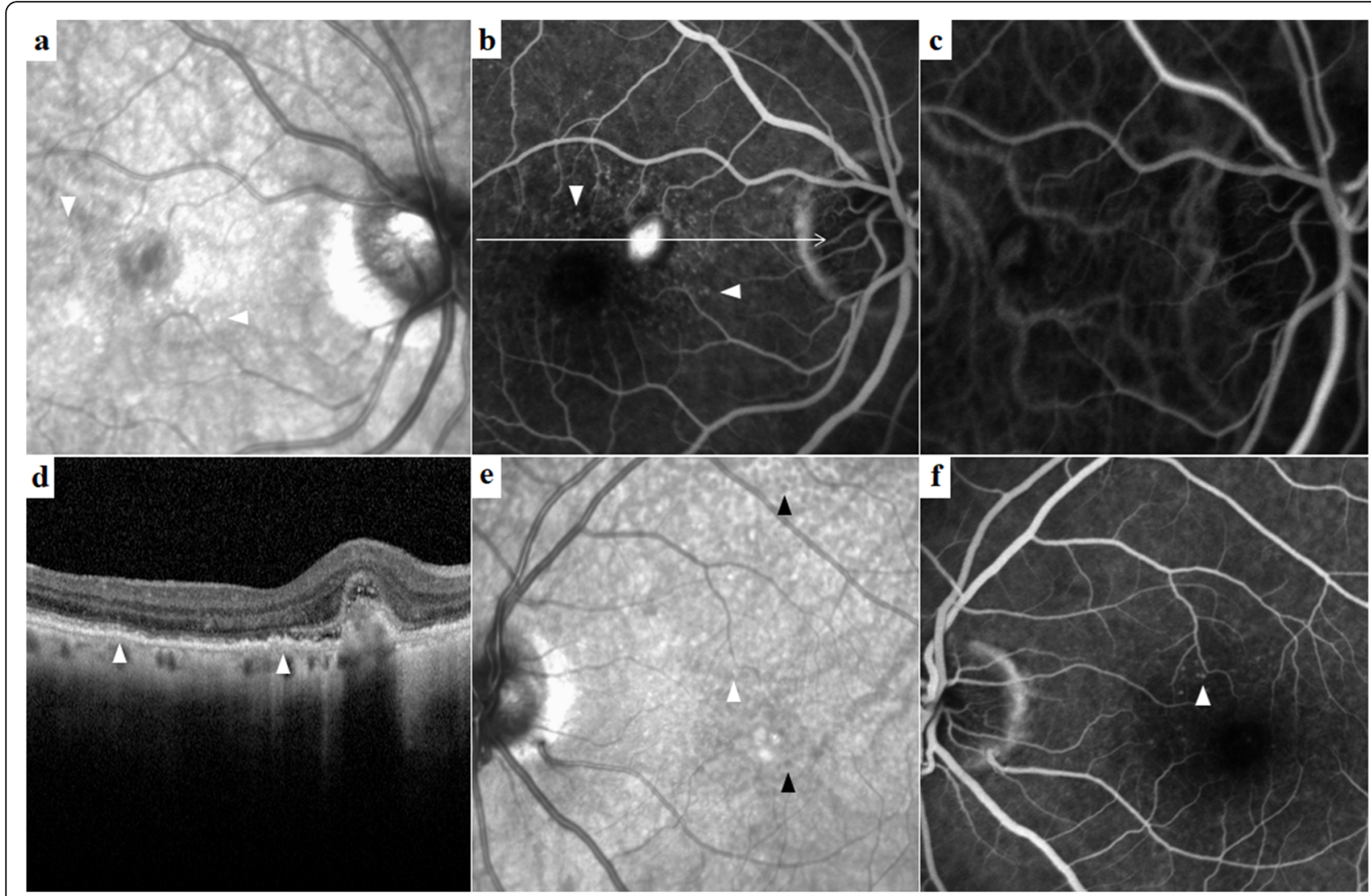

Fig. 1 Multimodal imaging of type 2 MNV, Drusen $\geq 63 \mu \mathrm{m}$ and SDD. a Drusen $\geq 63 \mu \mathrm{m}$ (arrowheads) displayed in IR. b FA with early MNV leakage and drusen staining (scan line). c ICGA revealed a circumscribed MNV besides choroidal vessels under speckled hypocyansecence. $\mathbf{d}$ SDOCT B-scan section through the lesion illustrated soft drusen (arrowheads) and a thin SFCT (109 $\mu \mathrm{m})$. e Drusen $>63$ (white arrowhead) and SDD (black arrowheads) visualized in the patient's fellow eye as demonstrated in IR and (f) soft drusen staining in late FA

and measure the size of retinal debris. Soft drusen (Fig. 1d), hard drusen or cuticular drusen (Fig. 2b, d) were identified as deposits below or within the RPE, whereas SDD were described as subretinal accumulation of material overlying the RPE zone, forming sharp, broad or rounded elevations [6]. A horizontal diameter of less than $63 \mu \mathrm{m}$ was defined as threshold to distinguish small from larger drusen. The Spectralis software allowed for a correlation of the topography in SD-OCT scans and $30^{\circ}$ en-face images. Drusen phenotypes, SDD, fibrosis or atrophy were identified and listed separately if coexistence occurred. Another senior clinical advisor without affiliation to the study concept was consulted in the case of grading disagreement.

SFCT was measured manually within SD-OCT scans as the greatest vertical distance between the RPE or the Bruch's membrane and the sclerochoroidal interface.

\section{Statistics}

A univariate logistic regression model was performed for each factor (gender, age, SFCT) potentially influencing the occurrence of drusen or SDD on the affected eye. The same analyses were performed to investigate the fellow eye. All calculations were executed using R, release 3.3.3. Diagrams were developed by Microsoft Excel (Microsoft Corporation, Redmond, WA) and figures composed by Photoshop CC 14.0 (Adobe Systems Incorporated, San Jose, CA).

\section{Results}

Data records of 835 eyes in 749 consecutive patients with nAMD were reviewed for MNV distribution. Type $1 \mathrm{MNV}$ was diagnosed in 658 eyes (78.8\%), type $2 \mathrm{MNV}$ in 27 eyes (3.2\%), type $3 \mathrm{MNV}$ in 75 eyes (9\%), mixed type $\mathrm{MNV}$ in 51 eyes $(6.1 \%)$ and polypoidal $\mathrm{MNV}$ in 24 eyes $(2.8 \%)$.

27 eyes of 27 patients with type $2 \mathrm{MNV}$ could be enrolled for further analysis. Type $2 \mathrm{MNV}$ was present in 11 (41\%) female and 16 (59\%) male patients with a mean age of $73.3 \pm 7.1$ standard deviation (SD) years (range: $57-86$ years). Overall, 17 eyes (63\%) compromised by type $2 \mathrm{MNV}$ presented drusen or SDD, leaving 10 eyes (37\%) without any extracellular debris (Fig. 3). Drusen or SDD were present in 14 of 23 fellow eyes (60.9\%). The distribution of drusen subtypes and their proportions was displayed in Table 1. Patients with drusen or SDD 


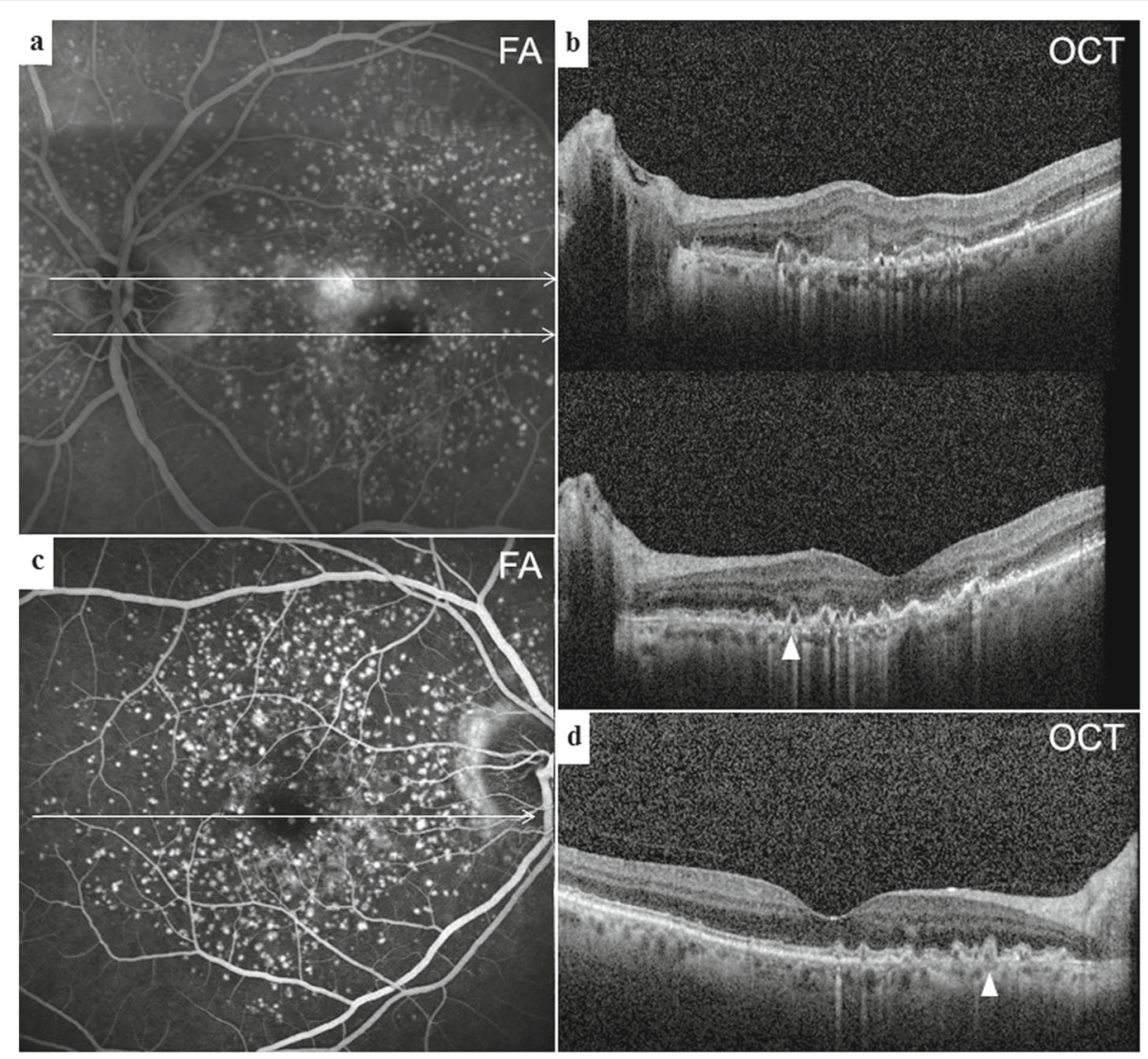

Fig. 2 Multimodal imaging of type 2 MNV and cuticular drusen. a FA with MNV leakage next to a hyperfluorescent "starry sky" distribution corresponding to the saw tooth configuration (arrowhead) of (b) SD-OCT B-scan sections (upper) through the lesion with some hyperreflective material in the subretinal space and (lower) through the foveal umbo. c FA demonstrated a similarly clustered posterior pole in the fellow eye with (d) multiple elevated pigment epithelium peaks in OCT B-scans typical for cuticular drusen (arrowhead)

in the neovascular eye were significantly younger (mean $70.88 \pm 6.85, p=0.04)$ than patients without deposits (mean $77.40 \pm 5.74$ ). Sex was not significantly associated with the appearance of drusen or SDD. The mean SFCT was $140 \pm 49 \mu \mathrm{m}$ in the neovascular eyes and $152 \pm$ $41 \mu \mathrm{m}$ in the fellow eyes. It had no significant impact on the occurrence of drusen or SDD. Extrafoveal atrophy was evident in 1 fellow eye (4.3\%). Four fellow eyes were excluded either due to neovascularization (1 eye with mixed type MNV) or fibrosis (3 eyes).

\section{Discussion}

In this retrospective cohort analysis of 835 eyes complicated by nAMD, only 27 (3.2\%) were compromised by type 2 MNV. Drusen or SDD - the hallmark of AMD were absent in a representative number of $10(37 \%)$ neovascular eyes and 9 (39.1\%) fellow eyes. Interestingly, age was the only accompanying factor in a substantial part of patients with pure type 2 lesions. Eyes compromised by this subtype and co-existing deposits were significantly related to younger age in contrast to eyes without additional signs of AMD. It is considered a typical neovascular subtype in entities such as high myopia, inflammatory chorioretinopathies or angioid streaks. Idiopathic choroidal neovascularization was originally described as a focal or type 2 lesion in patients younger than 50 years by $\mathrm{Hu}$ et al. in 1995 [30]. These findings suggest a more heterogenic picture of patients compromised by this phenotype of AMD.

Spaide recently proposed a new classification of AMD based on soft drusen, pachydrusen and SDD with predictive capabilities regarding a further development to late stage disease [8]. The appearance of soft drusen might lead to any type of MNV as well as GA. Eyes with SDD - particularly these with dot SDD - could progress to type 3 or type $2 \mathrm{MNV}$, whereas GA is typically associated with confluent SDD. We were not able to relate SDD subtypes to type $2 \mathrm{MNV}$. Only one other group focused primarily on clinical characteristics of type 2 lesions in nAMD [13]. 8 of 694 patients expressed this type of neovascularization with 7 of them (88\%) possessing SDD and thin choroids. Deposits must have been documented by multimodal imaging before the development of MNV. The authors concluded that the low 


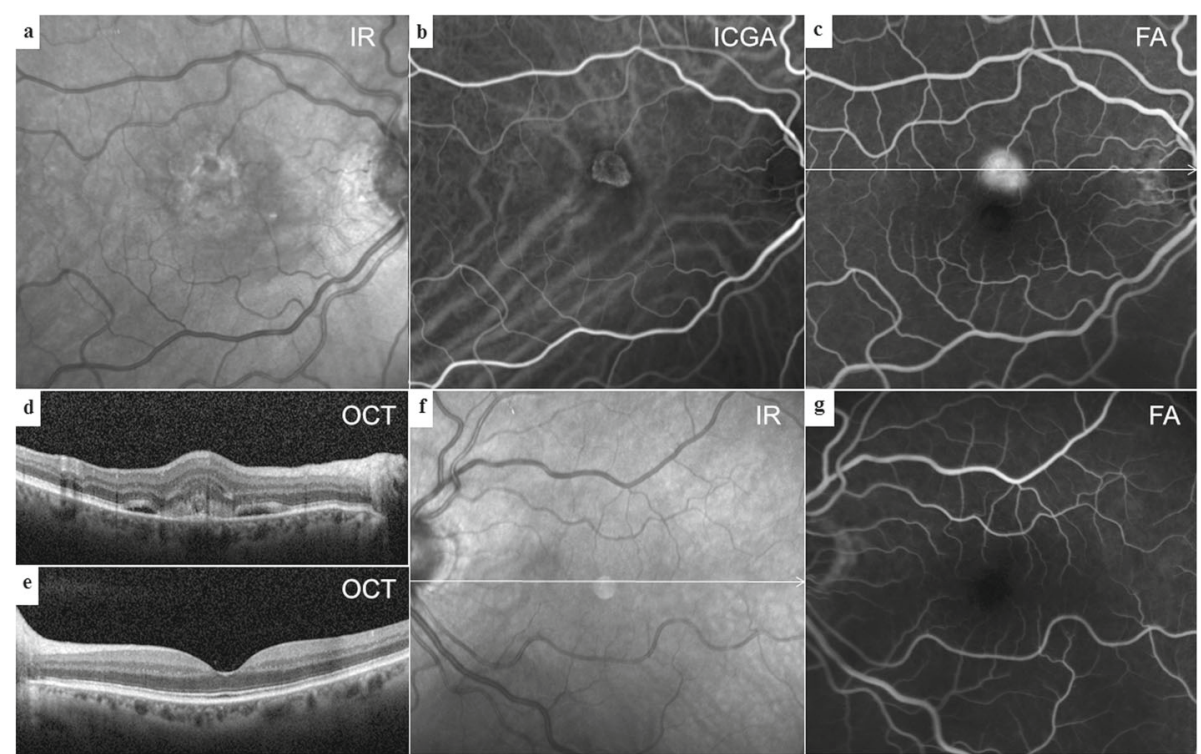

Fig. 3 Multimodal imaging of type 2 MNV without deposits. a Central hyperreflectivity surrounding a hyporeflective dot induced by the MNV lesion without evidence for drusen or subretinal drusenoid deposits in IR. b Early ICGA of the well-defined type 2 MNV. c Late FA showing MNV leakage (d) $30^{\circ}$ SD-OCT B-scan through the lesion with subretinal hyperreflective material next to fluid without outer retina irregularity. e No deposits in the fellow eye as demonstrated in a $30^{\circ}$ SD-OCT B-scan, (f) IR imaging or (g) FA

incidence of type $2 \mathrm{MNV}$ could be attributed to the strict inclusion criteria. Drusen regression with subsequent formation of MNV or GA has already been described [31, 32]. Also, SDD might regress and convert into atrophy over time [33]. We were looking for extracellular material at the onset of neovascular changes and initially excluded eyes with subfoveal atrophy. In concordance to our data, Wilde et al. found no difference in the occurrence of nAMD subtypes and SDD [34]. Another paper published by Marsiglia et al. could not correlate SDD with type $2 \mathrm{MNV}$ significantly 1 year earlier [35]. They investigated the association between MNV subtypes and clinical findings of the non-neovascular fellow eye in patients with unilateral nAMD. SDD and thin SFCT were more frequently seen in fellow eyes of patients with type $3 \mathrm{MNV}$, whereas type $1 \mathrm{MNV}$ was associated with a decreased odds ratio for SDD and a thin SFCT. We carefully monitored the SFCT of both the affected and the fellow eyes of patients with type $2 \mathrm{MNV}$ and compared eyes with drusen and SDD to eyes without retinal deposits. The term pachydrusen referred to pachychoroid according to Spaide and evolved preferentially into type $1 \mathrm{MNV}$ or polypoidal choroidal vasculopathy [8]. Choroidal thickness measured max. $259 \mu \mathrm{m}$ in eyes with type $2 \mathrm{MNV}$, as per definition pachychoroidassociated drusen could not be detected. Moreover,

Table 1 Distribution of characteristics in type 2 macular neovascularization

\begin{tabular}{lll}
\hline & Neovascular eyes & Non-neovascular fellow eyes \\
\hline No drusen & $10(37 \%)$ & $9(39.1 \%)$ \\
Drusen $<63 \mu \mathrm{m}$ & $2(7.4 \%)$ & $2(8.7 \%)$ \\
Drusen $\geq 63 \mu \mathrm{m}$ & $10(37 \%)$ & $9(39.1 \%)$ \\
Cuticular drusen & $2(7.4 \%)$ & $1(4.3 \%)$ \\
Subretinal drusenoid deposits (SDD) & $8(29.6 \%)$ & $5(21.7 \%)$ \\
Dot SDD & $3(11.1 \%)$ & $2(8.7 \%)$ \\
Ribbon SDD & $1(4 \%)$ & $1(4.3 \%)$ \\
Dot and ribbon SDD & $4(15 \%)$ & $2(8.7 \%)$ \\
Extrafoveal atrophy & $0(0 \%)$ & $1(4.3 \%)$ \\
Subfoveal atrophy & $0(0 \%)$ & $0(0 \%)$ \\
Fibrosis & $0(0 \%)$ & $3(13 \%)$ \\
Total number of eyes & 27 & 23 \\
\hline
\end{tabular}


SFCT was not significantly related to extracellular material in the fellow eye. SDD were classified as visible in OCT B-scans but no significant pattern could be assessed in either eye. Drusen or SDD were absent in 9 of 23 fellow eyes (39.1\%). Abugreen et al. related neovascular subtypes to AMD severity of the fellow eye and found comparable numbers [36]. No features of AMD were exhibited in 10 of 23 fellow eyes (43.5\%) in patients compromised by classic lesions in 2003.

Limitations of this study include the retrospective evaluation of deposits in previously acquired images and their available information. Peripheral SDD or drusen could have been missed as the primary focus of interest was the macular area pictured by Heidelberg's Spectralis $30^{\circ}$ images. Certain questionnaires would be more likely to be answered in a prospective study design. Other MNV subtypes were not analyzed in detail, which impaired the study's comparative nature. On the other hand, MNV anatomy was examined by multimodal imaging in a large cohort of 835 consecutive patients. Twenty-seven eyes with type $2 \mathrm{MNV}$ in AMD are considered as this study's strengths. Two thirds of type 2 MNV were found in the presence of soft or hard drusen and SDD. One third of eyes without deposits was frequently related to older age, suggesting a more heterogenic picture of this phenotype.

\section{Conclusions}

The herein presented data contribute to a better understanding of the anatomical features in this rarely found nAMD subtype. AMD is not necessarily confined to the existence of extracellular deposits. Other biological pathways in AMD may lead to the development of a neovascularization limited to the subretinal space.

\section{Abbreviations \\ MNV: Macular neovascularization; AMD: Age-related macular degeneration; SFCT: Subfoveal choroidal thickness; SDD: Subretinal drusenoid deposits; RPE: Retinal pigment epithelium; nAMD: Neovascular AMD; GA: Geographic atrophy; IR: Infrared fundus reflectance; BAF: Blue-peak fundus autofluorescence; SD: Spectral-domain; OCT: Optical coherence tomography;" FA: Fluorescein angiography; ICGA: Indocyanine green angiography; IMNV: Idiopathic macular neovascularization}

\section{Acknowledgments}

Ilse Krebs, MD, a senior clinical advisor without affiliation to the study concept, was consulted in case of grading disagreement. The computing and post-processing of the figures were supported by Senior Clinical Advisor Carl Glittenberg, a Topcon employee.

\section{Authors' contributions}

DA, MS and SA-S contributed to the conception and design of the study. Acquisition of data was performed by DA, MS and A-MH. Analysis and interpretation of data was conducted by AG, DA and MS. DA, MS and A-MH drafted the article. KK and SA-S revised it critically for important intellectual content and approved the final version for publication. All authors read and approved the final version of the manuscript. All auhors have agreed to be personally accountable for the author's own contributions and ensured that questions related to the accuracy or integrity of any part of the work, even ones in which the author was not personally involved, were appropriately investigated, resolved, and the resolution documented in the literature.

\section{Funding}

No funding was received for this study.

\section{Availability of data and materials}

The datasets used and/or analyzed during the current study are available from the corresponding author on reasonable request.

\section{Ethics approval and consent to participate}

The Federal Hospitals Act §15a Abs. 3a states that a vote from the Viennese ethics committee is not obligatory for this study design. In the medical retina unit of the Rudolf Foundation Hospital, patients sign a waiver as part of their informed consent form for treatment or diagnostics indicating that anonymized clinical data may be used for scientific research unless patients specifically opted not to do so.

\section{Consent for publication}

Not applicable.

\section{Competing interests}

The authors declare that they have no competing interests.

\section{Author details}

${ }^{1}$ Karl Landsteiner Institute for Retinal Research and Imaging, Vienna, Austria. ${ }^{2}$ Department of Ophthalmology, Rudolf Foundation Hospital, Juchgasse 25, 1030 Vienna, Austria. ${ }^{3}$ Center for Medical Statistic, Informatics, and Intelligent Systems, Medical University of Vienna, Spitalgasse 23, 1090 Vienna, Austria. ${ }^{4}$ Department of Ophthalmology, Medical University of Graz,

Auenbruggerplatz 1, 8036 Graz, Austria.

Received: 6 August 2019 Accepted: 17 September 2020

Published online: 25 September 2020

\section{References}

1. Age-Related Eye Disease Study Research Group. A randomized, placebocontrolled, clinical trial of high-dose supplementation with vitamins $C$ and E, beta carotene, and zinc for age-related macular degeneration and vision loss: AREDS report no. 8. Arch Ophthalmol. 2001;119:1417-36.

2. Ferris FL, Wilkinson CP, Bird A, Chakravarthy U, Chew E, Csaky K, et al. Clinical classification of age-related macular degeneration. Ophthalmology. 2013:120:844-51.

3. Finger RP, Chong E, McGuinness MB, Robman LD, Aung KZ, Giles G, et al. Reticular Pseudodrusen and their association with age-related macular degeneration: the Melbourne collaborative cohort study. Ophthalmology. 2016:123:599-608.

4. Zweifel SA, Spaide RF, Curcio CA, Malek G, Imamura Y. Reticular pseudodrusen are subretinal drusenoid deposits. Ophthalmology. 2010;117: 303-312.e1.

5. Zweifel SA, Imamura Y, Spaide TC, Fujiwara T, Spaide RF. Prevalence and significance of subretinal drusenoid deposits (reticular pseudodrusen) in age-related macular degeneration. Ophthalmology. 2010;117:1775-81.

6. Spaide RF, Curcio CA. Drusen characterization with multimodal imaging. Retina (Philadelphia, Pa). 2010;30:1441-54.

7. Khan KN, Mahroo OA, Khan RS, Mohamed MD, McKibbin M, Bird A, et al. Differentiating drusen: Drusen and drusen-like appearances associated with ageing, age-related macular degeneration, inherited eye disease and other pathological processes. Prog Retin Eye Res. 2016;53:70-106.

8. Spaide RF, IMPROVING THE, AGE-RELATED

MACULARDEGENERATIONCONSTRUCT. A new classification system. Retina (Philadelphia, Pa). 2017.

9. Spaide RF, Jaffe GJ, Sarraf D, Freund KB, Sadda SR, Staurenghi G, et al. Consensus nomenclature for reporting Neovascular age-related macular degeneration data: consensus on Neovascular age-related macular degeneration nomenclature study group. Ophthalmology. 2020;127:616-36.

10. Dansingani KK, Gal-Or O, Sadda SR, Yannuzzi LA, Freund KB. Understanding aneurysmal type 1 neovascularization (polypoidal choroidal vasculopathy): a lesson in the taxonomy of "expanded spectra" - a review. Clin Exp Ophthalmol. 2018;46:189-200. 
11. Freund KB, Zweifel SA, Engelbert M. Do we need a new classification for choroidal neovascularization in age-related macular degeneration? Retina (Philadelphia, Pa). 2010;30:1333-49.

12. Jung JJ, Chen CY, Mrejen S, Gallego-Pinazo R, Xu L, Marsiglia M, et al. The incidence of neovascular subtypes in newly diagnosed neovascular age-related macular degeneration. Am J Ophthalmol. 2014;158:769-779. e2.

13. Naysan J, Jung JJ, Dansingani KK, Balaratnasingam C, Freund KB. Type 2 (subretinal) neovascularization in age-related macular degeneration associated with pure reticular pseudodrusen phenotype. Retina (Philadelphia, Pa). 2016;36:449-57.

14. Klein R, Klein BEK, Knudtson MD, Meuer SM, Swift M, Gangnon RE. Fifteenyear cumulative incidence of age-related macular degeneration: the beaver dam eye study. Ophthalmology. 2007;114:253-62.

15. Silva R, Cachulo ML, Fonseca P, Bernardes R, Nunes S, Vilhena N, et al. Agerelated macular degeneration and risk factors for the development of choroidal neovascularisation in the fellow eye: a 3-year follow-up study. Ophthalmologica. 2011;226:110-8.

16. Abdelfattah NS, Zhang H, Boyer DS, Rosenfeld PJ, Feuer WJ, Gregori G, et al. Drusen volume as a predictor of disease progression in patients with late age-related macular degeneration in the fellow eye. Invest Ophthalmol Vis Sci. 2016;57:1839-46.

17. George S, Cooke C, Chakravarthy U. Exudative AMD subtypes and eligibility for treatment with ranibizumab. Eye (Lond). 2010;24:1247-51.

18. Cohen SY, Creuzot-Garcher C, Darmon J, Desmettre T, Korobelnik JF, Levrat $F$, et al. Types of choroidal neovascularisation in newly diagnosed exudative age-related macular degeneration. $\mathrm{Br} J$ Ophthalmol. 2007;91:1173-6.

19. Olsen TW, Feng $X$, Kasper TJ, Rath PP, Steuer ER. Fluorescein angiographic lesion type frequency in neovascular age-related macular degeneration. Ophthalmology. 2004;111:250-5.

20. Freund KB, Yannuzzi LA, Sorenson JA. Age-related macular degeneration and choroidal neovascularization. Am J Ophthalmol. 1993;115:786-91.

21. Suzuki M, Sato T, Spaide RF. Pseudodrusen subtypes as delineated by multimodal imaging of the fundus. Am J Ophthalmol. 2014;157:100512.

22. Balaratnasingam C, Cherepanoff $\mathrm{S}$, Dolz-Marco R, Killingsworth $M$, Chen FK, Mendis R, et al. Cuticular Drusen: clinical phenotypes and natural history defined using multimodal imaging. Ophthalmology. 2018;125: 100-18.

23. Delori FC, Fleckner MR, Goger DG, Weiter JJ, Dorey CK. Autofluorescence distribution associated with drusen in age-related macular degeneration. Invest Ophthalmol Vis Sci. 2000;41:496-504.

24. Smith RT, Chan JK, Busuoic M, Sivagnanavel V, Bird AC, Chong NV. Autofluorescence characteristics of early, atrophic, and high-risk fellow eyes in age-related macular degeneration. Invest Ophthalmol Vis Sci. 2006;47: 5495-504.

25. Pauleikhoff D, Zuels S, Sheraidah GS, Marshall J, Wessing A, Bird AC Correlation between biochemical composition and fluorescein binding of deposits in Bruch's membrane. Ophthalmology. 1992;99:1548-53.

26. Arnold JJ, Quaranta M, Soubrane G, Sarks SH, Coscas G. Indocyanine green angiography of drusen. Am J Ophthalmol. 1997;124:344-56.

27. Prenner JL, Rosenblatt BJ, Tolentino MJ, Ying G-S, Javornik NB, Maguire MG, et al. Risk factors for choroidal neovascularization and vision loss in the fellow eye study of CNVPT. Retina (Philadelphia, Pa). 2003;23:307-14.

28. Mimoun G, Soubrane G, Coscas G. Macular drusen. J Fr Ophtalmol. 1990;13: 511-30.

29. Gass JDM. Stereoscopic atlas of macular diseases: diagnosis and treatment. 2d ed. St. Louis: C. V. Mosby; 1977.

30. Ho AC, Yannuzzi LA, Pisicano K, DeRosa J. The natural history of idiopathic subfoveal choroidal neovascularization. Ophthalmology. 1995; 102:782-9.

31. Cukras C, Agrón E, Klein ML, Ferris FL, Chew EY, Gensler G, et al. Natural history of Drusenoid pigment epithelial detachment in age-related macular degeneration: AREDS report number 28. Ophthalmology. 2010;117:489-99.

32. Yehoshua Z, Wang F, Rosenfeld PJ, Penha FM, Feuer WJ, Gregori G. Natural history of drusen morphology in age-related macular degeneration using spectral domain optical coherence tomography. Ophthalmology. 2011;118: 2434-41.
33. Spaide RF, Ooto S, Curcio CA. Subretinal drusenoid deposits AKA pseudodrusen. Surv Ophthalmol. 2018;63:782-815.

34. Wilde C, Patel M, Lakshmanan A, Morales MA, Dhar-Munshi S, Amoaku WMK. Prevalence of reticular pseudodrusen in eyes with newly presenting neovascular age-related macular degeneration. Eur J Ophthalmol. 2016;26: 128-34.

35. Marsiglia M, Boddu S, Chen CY, Jung JJ, Mrejen S, Gallego-Pinazo R, et al. Correlation between neovascular lesion type and clinical characteristics of nonneovascular fellow eyes in patients with unilateral, neovascular age-related macular degeneration. Retina (Philadelphia, Pa). 2015;35:966-74

36. Abugreen S, Muldrew KA, Stevenson MR, VanLeeuwen R, DeJong PTVM, Chakravarthy U. CNV subtype in first eyes predicts severity of ARM in fellow eyes. Br J Ophthalmol. 2003;87:307-11.

\section{Publisher's Note}

Springer Nature remains neutral with regard to jurisdictional claims in published maps and institutional affiliations.
Ready to submit your research? Choose BMC and benefit from:

- fast, convenient online submission

- thorough peer review by experienced researchers in your field

- rapid publication on acceptance

- support for research data, including large and complex data types

- gold Open Access which fosters wider collaboration and increased citations

- maximum visibility for your research: over $100 \mathrm{M}$ website views per year

At BMC, research is always in progress.

Learn more biomedcentral.com/submissions 